"Talk to Us". Since this time two further CCGs have joined the project.

The principle behind Talk to Us is very simple, patients or bereaved carers who have self-nominated talk to volunteers who record their experiences. The conversation is led by the participant; it really is about their experience. Once the conversation has been concluded the volunteers think about what they have heard and how it relates to the Quality Standards for end of life care for adults (QS13). The role of the volunteers should not be underestimated as it is their careful support that draws out the experiences without the use of questions that might risk influencing the direction of the conversation.

The written account is used by the CCG Quality Lead to inform and influence system wide improvement. The challenge has been to retain the narrative as this is where the impact lies. Sharing the narratives has ignited change where data would have had little impact.

29 recorded experiences which have resulted in action will be discussed at a Stakeholder event in June 2016 prior to the publication of an impact report.

\section{P-153 WHAT DO PATIENTS, CARERS AND FAMILY MEMBERS VALUE FROM HOSPICE SERVICES?}

${ }^{1}$ Nicole Hughes, 'Lindsay Eckley, 'Jane Noyes, ${ }^{2}$ Trystan Pritchard. 'Bangor University, Bangor, UK; ${ }^{2}$ St David's Hospice

\subsection{6/bmjspcare-2016-001245.176}

Background Within the UK, over a quarter of a million people currently receive care from hospices either within hospice care facilities or within their own homes. In a difficult financial climate and uncertain funding support, independent Hospices need to reaffirm their impact and contribution to society. However, there is limited information on what outcomes patients, carers and families view as important. Here, we present findings from a systematic literature review collating the evidence.

Aims A systematic literature review was conducted to explore patients', carers' and family members' experiences and perceptions of hospice services

Methods

- Literature searching

Key terms and Medical Subject Headings were implemented in a range of databases to identify studies and grey literature.

\section{- Study selection}

Titles and abstracts were screened against a criteria list and papers were chosen based on relevance to the research question.

\section{- Quality assessment}

Papers were assessed for quality using appropriate tools

\section{- Data extraction and collation}

Data from each included paper was fully screened, recorded and summarised

Results Pending as review still being undertaken at time of abstract submission

Wider study This review is part of a study funded by Knowledge Economy Skills Scholarship, a major European Convergence programme which offers collaborative research projects linked with a local company partner. The aim of the research is to explore the social value of hospices in North Wales. The Social Return on Investment framework will be utilised to quantify social and environmental values allowing for an understanding which extends beyond financial values from key stakeholder perspectives. A combination of qualitative narratives and quantitative measurements will be utilised including:

- Semi-structured interviews with staff

- Review of patient feedback

- Analysis of current hospice data

- Searching unit cost and social cost databases to source proxy values for outcomes.

\section{P-154 DEVELOPING A METHODOLOGY TO GATHER FEEDBACK FROM PEOPLE ACCESSING SPECIALIST COMMUNITY PALLIATIVE CARE}

Libby Milton, Ben Gadd, Anne Finucane. Marie Curie

\subsection{6/bmjspcare-2016-001245.177}

Understanding the experiences of people who use a service is crucial to support quality improvement, however, gathering unbiased feedback is particularly challenging when the service is delivered in the person's own home, when they may be dying or distressed.

The aim of this project was to develop an effective and sustainable approach to gathering feedback from both patients and carers about their experiences accessing a specialist community palliative care service.

Such feedback will i) inform new service development, ii) provide quality assurance to the team, managers and regulators and iii) provide RNs evidence for revalidation of their registration What is being done and how it is being evaluated

A workshop with key stakeholders explored the purpose of the survey and key measures, based on the Warwick Patient Experience framework (Staniszewska et al., 2014). Questions were prioritised, recognising the link between length of survey and response quality (Cape, 2010). Selecting questions that were validated, or heavily utilised in other healthcare settings, using Likert-style ratings and free text response options and following feedback from our patient and public involvement group, the questions were tested on a sample of patients and carers before rolling out.

Questionnaires are sent both to community patients and to family/carers following their bereavement. Response rate for the first four months was 28\% ( $\mathrm{n}=108)$.

Overall satisfaction rating is $95 \%$, with the highest response reporting perceived dignity at $98.4 \%$ and the lowest around support for whole person, being $89 \%$.

The methodology appears to successfully provide a mechanism for experience of care reporting in this setting. A procedure was developed to follow up negative comment directly with the respondent, or, if anonymous, within a team reflection session, demonstrating a commitment to quality improvement. Positive comments are circulated.

Feedback received thus far demonstrates the effectiveness of the approach, and is proving to be a valuable contribution to quality assurance and service development. 